\title{
Approaches to antiretroviral therapy in China
}

\author{
Bruce L GILLIAM*, Robert R REDFIELD \\ Division of Clinical Care and Research, Institute of Human Virology, University of Maryland, Baltimore, MD 21201, USA
}

\begin{abstract}
China has recognized the threat of HIV to its population and responded with a national antiretroviral treatment (ART) program. However, high ART failure rates and the spread of resistance within populations are important realities to consider when developing and managing ART programs in China and worldwide. Concepts which will define treatment success and local and national programmatic goals are 1) access to ART, 2) durability of ART at the patient level, 3) scalability of treatment modalities, and the 4) sustainability of the program at the community or national level. In the face of limited resources, China must also consider when to start ARV therapy, which agents to use, when to switch them, and how to treat highly experienced patients with drug resistance. The optimal ARV regimen to start with is changing frequently with the introduction of new agents and the presentation of new data. Currently, a regimen including tenofovir, emtricitabine or lamivudine and a nonnucleoside reverse transcriptase inhibitor appears to have optimal characteristics to treat HIV/AIDS in China. However, critical to all of these choices is the evaluation of programs implemented to insure wide scale success. China has wisely begun this process of evaluating the performance of local programs through systematic monitoring and evaluation of treatment outcomes. This will allow regimens and programs that work to be expanded, and programs with high failure rates to be eliminated. In the end, evidence based data supporting treatment strategies will allow China to successfully confront its AIDS epidemic early and prevent its tragic consequences
\end{abstract}

Keywords: antiretroviral therapy, scalability, HIV/AIDS.

\section{INTRODUCTION}

Advances in antiretroviral therapy (ART) have resulted in dramatic declines in hospitalizations and death rates in the U.S. and Europe, with concomitant steady increases in life expectancy. In China, the government has recognized the imminent threat that HIV/AIDS poses to its population and responded with a national antiretroviral (ARV) treatment program providing ARV drugs free to those most in need [1]. However, given the potentially catastrophic impact of HIV and the transmissibility of drug resistant HIV strains, the development and implementation of sustainable and affordable ART strategies is a priority for China.

It is important to recognize that despite the significance of the recently implemented ARV treatment program in China, access to the ARV drugs is only the tip of the iceberg. Key issues must be clearly defined and addressed for a

\footnotetext{
*Correspondence: Bruce L GILLIAM

725 W. Lombard St.Baltimore, MD, USA 21201

Tel: 410-706-4613; Fax: 410-706-1992

E-mail: gilliam@umbi.umd.edu
}

larger and diverse country such as China to establish and implement a successful ART program. Examples include: 1) the development of drug procurement and delivery systems; 2) augmentation of expertise in the management of ART; 3) enhancement of care and treatment infrastructure particularly in regions where the epidemic is entrenched; 4) development of alternative treatment delivery models which lend themselves to rapid scalability; 5) define and validate the safety and efficacy of the optimal ARV regimens for use in China; 6) establishment of monitoring and evaluation capacity to ensure success in ART approaches in different settings and populations throughout China.

\section{KEY CONCEPTS FOR IMPLEMENTATION OF A CHINESE ARV PROGRAM: ACCESS, DURABILITY, SCALABILITY AND SUSTAI- NABILITY}

The treatment of HIV/AIDS has advanced tremendously over the past two decades and many patients may now be able to live a normal life span if treated effectively. Much 
has been learned in the US and Europe about the limitations of effective ART. Recent clinical trials of new ARV agents have demonstrated astounding success rates with $>70 \%$ of individuals treated attaining durable viral suppression [2-5]. However, the success rates in real life clinic settings have been much lower [6]. This has led to an increase in the prevalence of resistance mutations in both those on ART and those who are newly infected $[7,8]$. Indeed, a report in this issue demonstrates that $62.7 \%$ of patients developed ARV drug resistance after only 6 months of ART in one region in China [9]. These high failure rates and the spread of resistance within populations are important realities to consider when developing and managing ART programs in China and worldwide. The proactive prevention of the emergence of HIV and Hepatitis B (HBV) resistance to ARV drugs in both the individual patient and the community is extremely important. How successfully this can be accomplished will determine the long term sustainability of newly initiated national ARV programs.

Concepts which will define treatment success and local and national programmatic goals are 1) access to ART, 2) durability of ART at the patient level, 3) scalability of treatment modalities, and the 4) sustainability of the program at the community or national level. Central to each of these strategic variables is the definition of programmatic success.

Access to treatment is the ability of individuals or communities to obtain not only ART but also appropriate medical care and management. Access to the medical care needed to administer high quality ART is also a major hurdle to the delivery of ART in many parts of China. Once ART is finally obtained, there is then the challenge of receiving appropriate management of these complex treatments including drug toxicities, drug-drug interactions, adherence, and resistance. Despite only approximately 1 million patients on therapy in the U.S. and Europe, access to ARVs and to medical care with expertise in HIV treatment is still a recognized problem. Even in the U.S., many individual states have waiting lists to obtain recommended ART.

Durability of treatment can be defined as treatments which produce the desired effect, for example viral suppression, for an extended period of time. It is hoped that this period of time would be measured in decades, so that individuals can live a natural lifetime. The ramifications of a non-durable initial ART regimen include disease progression, opportunistic infections, ARV drug resistance, and expanded diagnostic monitoring (such as frequent viral load and viral resistance testing) as required components of routine clinical care. If only one primary ART regimen is available, failure of that regimen may lead to death for an individual who has no further options. In addition, resistant virus may be transmitted, leading to lack of efficacy of the one primary regimen in ARV naive patients. This would, in turn, limit therapeutic options for all HIV infected individuals in China.

Key variables to improve the durability of therapeutic response include the choice of regimen, the care delivery system and the therapeutic strategy used. The choice of ARV drugs can directly impact durability of a regimen due to a number of properties of the drugs including their toxicity/side effects, mutation threshold of the drugs either alone or in combination, $\mathrm{pK}$ profiles and drug-drug interactions. Studies have demonstrated that care delivery systems such as directly observed therapy can have a huge impact on the durability of an ARV regimen [10-12]. Additionally, the therapeutic strategy used may play a role in durability by utilizing ARVs under conditions which optimize pharmacokinetics and other drug characteristics. An example of this are induction-maintenance strategies which seek to optimize viral suppression early with multiple high mutation threshold drugs and then de-intensify to simple, non-toxic drugs for long-term maintenance.

Once access to a durable ART regimen has been established, the ability of HIV treatment strategies to be "scaled up" to allow the treatment of all HIV infected individuals in a prefectorate, province or nationwide is critical to the long term success of the management of HIV disease. This can be termed scalability $[13,14]$. There are a number of variables which will determine the scalability of a selected ARV program. First, the selection of the regimen will dictate capacity to scale. For example, regimens that do not require laboratory testing for toxicity monitoring will be easier to bring to scale. Second, the ART model selected will dictate capacity to scale. For example, hospital or clinic based ARV programs will have limitations. Village, workplace, or parish centered ARV treatment programs lend themselves to rapid expansion. Third, systems needed to maintain an adequate supply of the necessary ARVs, need to be in place. Substantial logistical management is required to ensure that the drug supply is maintained without interruption and that the drug is utilized prior to expiration. Fourth, development and implementation of strong community based treatment education and preparation programs that proactively engage the community in participating in a program promoting early diagnosis, early treatment and support of persons living with HIV infection will be crucial. Without it, many, who on paper have access to HIV drugs, may not actually be able to obtain treatment due to lack of infrastructure, the need for specialized lab tests, the need for a certain level of education to administer the drugs, the lack of facilities to store temperature sensitive drugs, or the cost limitations of providing care which often far exceed the cost of the ARV medications. Each of these concepts is hopelessly inter- 
twined and equally important in the consideration of ART in a prefectorate or province. Overall, it is important that from the initial vision of a national ARV program, these issues related to scalability are thought through.

After an ARV program has been brought to scale and is able to provide ART to the people living with HIV in the community, it will become critical to sustain this capacity in order for the ARV program to negate the impact of HIV on its society. Two major prerequisites will be required for long term sustainability. First, the development of ARV resistance has been successfully minimized during the course of implementation of the ARV program, so that the selected ARV regimen still retain its antiviral efficacy once the program is scaled. Second, sustainability will be dependent on the continued support of the government and donor agencies to underwrite the cost of ART. These programs will need to be effective in terms of globalization and relevant economic, social and humanitarian concerns to justify a sustained level of support.

The strategies created in China to deliver ART are developed with the goal of "success". However, success is determined by one's outlook. For example, Brazil's experience implementing ART has been proclaimed as a success by some who point out the tremendous access to ARVs created by the program and the declining mortality rate of HIV-infected individuals since the implementation of ART. However, others will highlight that, in a short period of time, $6 \%$ of newly diagnosed and more than $40 \%$ of ARV treated patients in Brazil have evidence of resistance $[15,16]$. They suggest that this is what has led to Brazil's increased ARV costs and will lead to future increased mortality. In fact, Brazil stated that $25 \%$ of its overall health budget is now spent on purchasing Kaletra, an ARV used as a second/third line drug in the Brazilian ARV program [17]. Therefore, the goals of a therapeutic strategy, such as viral suppression over time or percent drug resistance, must first be defined to determine the success or failure of that strategy and insure wide scale implementation of a successful treatment strategy.

\section{KEY PRINCIPLES AND COMPLEXITIES OF ANTIRETROVIRAL THERAPY}

The appropriate management of ART is a continually evolving process which should change as new data becomes available. When considering selection of an ARV regimen one must synthesize the issues of viral dynamics and pathogenesis [18-22], resistance, drug pharmacokinetics, drug toxicities, and drug durability. It is also essential to learn from the hard-learned lessons of the past 15-20 years of ART. It is now clear that sequential mono or dual therapy as occurred with ZDV and/or didanosine (ddI) therapy, implemented and scaled up without an ad- equate understanding of the importance of adherence, resistance, pharmacokinetics, and provider experience, led to resistance and limitation of future treatment options. In retrospect, it may have been more prudent to optimize and validate issues related to ARV durability before wide scale implementation of ART recommendations. Although unintended, these early decisions served to enable viral resistance which further complicated ART. For example, a high prevalence of resistance in patients with primary or early HIV infection can inadvertently lead to monotherapy in a newly treated patient if resistance exists to one or two of the drugs chosen for that person's initial ART regimen. Wide scale non nucleoside reverse transcriptase inhibitor (NNRTI) resistance induced by "successful" MTCT programs could significantly negatively impact the success of a National ARV program utilizing an NNRTI containing ARV regimen. These are just examples of how early decisions may impact future ARV program options and add to the complexity of ART management. China has wisely chosen to investigate these critical issues by carefully monitoring groups initiated early on ARV regimens and determining their treatment outcomes. This decision may help China to avoid the large scale negative outcomes seen in those treated in the U.S. and Europe during the first decade of ARV treatment.

In the face of limited resources, China must consider when to start ARV therapy, which agents to use out of the 21 drugs and multiple preparations available worldwide, when to switch them, and how to treat highly experienced patients with drug resistance. The decision of when to start ART in an individual is a much debated question worldwide without a clear answer, as evidenced by the United States and Great Britain choosing different criterion despite evaluating the same data. There is consensus that therapy should be initiated when a patient develops an opportunistic infection or has CD4 cell count $<200$ [23]. Above this, there is a debate over the optimal starting CD4 cell count [24-26]. Data indicates a much higher morbidity and mortality rate if started at CD $4<200$. However, above this level, the benefit of therapy to prevent disease progression is smaller, although ART has been demonstrated to have a greater efficacy and lower toxicity at higher CD4 cell counts. In addition, in countries such as China where TB remains an active public health problem, delaying the initiation of ARV to patients with advanced immunodeficiency may have significant implications related to TB transmission. In HIV infected patients co-infected with $\mathrm{TB}, \mathrm{TB}$ reactivation often occurs at $\mathrm{CD} 4$ counts between 200-400. If ARV intervention is delayed, an opportunity to limit the spread of TB may be missed along with the prevention of isolated TB disease in the individual patient. China has chosen to carefully evaluate the data 
available and develop a Chinese staging system to direct these decisions. This system recommends consideration of treatment for those individuals with clinical immunosuppression, opportunistic infections and CD4 < 200, and sub-clinical immunosuppression, CD4 $<350$. Also, all HIV patients with TB should be considered for ART independent of underlying immune status.

The optimal ARV regimen to start with is changing frequently with the introduction of new agents and the presentation of new data. The basic selection criteria for an ARV regimen include: high potency, high threshold of resistance, low toxicity, favorable pharmacokinetics (i.e., once or twice daily administration, co-formulation), similar drug half-lives amongst drugs used, equal activity in different cellular growth cycles, few short term and long term side effects and few drug interactions, particularly with TB medications. These criteria have led to changes in primary treatment regimens in the U.S. and Europe. Recognition of the limitations of the thymidine analogs, stavudine (d4T) and ZDV, have prompted clinicians to replace these agents with potent, once daily, low toxicity agents such as tenofovir (TDF) and abacavir(ABC). Lowering the potential to develop drug toxicities is an extremely important issue when ARV programs select one regimen. Choice of regimens which may include thymidine analogs $[27,28]$ and nevirapine (NVP) [29] must be balanced by accumulating toxicities of long term therapy, increased cost, and potential for development of resistance prior to onset of symptomatic AIDS. This is highlighted by the $62.7 \%$ resistance seen after 6 months of ZDV/ddI/NVP in China [9]. In addition, the presence of co-morbidities such as HBV must also be factored into the selection of antiretroviral agents, especially given the high prevalence of HBV in China. Therefore, consideration should be given to the inclusion of antiretroviral agents with HBV activity, TDF, lamivudine (3TC) and emtricitabine (FTC), in an HIV treatment regimen and the avoidance of HBV monotherapy with $3 \mathrm{TC}$ alone in order to prevent HBV resistance.

HIV management also includes dealing with the toxicities of these drugs [27, 28, 30-33], drug-drug interactions, difficulties with the need for $100 \%$ adherence [34], lack of long-term efficacy of regimens in real life settings [3539], cost, co-morbid illnesses such as hepatitis and tuberculosis, opportunistic infections and their prophylaxis, substance abuse, mental illness, and the social situations of patients (housing, nutrition, stigma, family/reproductive counseling, secondary prevention, etc.). Adding to the complexity is the fact that there is no one answer for any of these issues. It is therefore not a surprise that numerous studies have demonstrated that the more experience a provider has in the care of HIV infected patients the better the outcomes in terms of viral suppression, disease progression, and death. New treatment strategies built on the hard learned lessons from existing ARV programs can and should be developed in order to avoid the mistakes of the past.

Consideration of switching or changing ART is given when an individual does not have an appropriate initial response to therapy, has an increase in viral load following complete viral suppression, and has a decreasing CD4 cell count, and/or toxicity or intolerability to therapy. Antiviral drug resistance and ARV side effects are the major causing of switching therapy. Resistance has become a significant problem in the United States, Europe and, more recently Brazil. The high replication rate and low replication fidelity (high error rate) of HIV predisposes to the development of resistance. In general, resistance develops on ART in the face of ongoing viral replication and significant resistance can be occurring despite a stable or increasing CD4 count. Thus, treatment strategies which rely on CD4 counts alone to determine treatment failure will likely see patients with accumulated resistance mutations at the time of recognized clinical failure. Some data also suggests that resistance may occur when initiating therapy and prior to complete viral suppression. This can be problematic when considering agents with a low mutation threshold such as 3TC, efavirenz (EFV), and NVP. These agents have been chosen in developing countries due to their ease of administration, costs, and potency. Resistance to EFV and NVP has been shown to develop rapidly in clinical use and has been documented to occur in up to $15 \%$ of patients with primary/early HIV infection and no history of exposure to antiretroviral therapy [7]. In addition, we are recognizing the fact that high rates of resistance to NNRTIs occur after single dose NVP is given for PMTCT [40]. It is now clear that significant cross-resistance occurs within each of the antiretroviral drug classes. Use of one or two agents in a given class may lead to resistance to the entire class of agents. Stated another way, if resistance mutations to the initial ART regimen occur and are allowed to accumulate, future treatment options may be extremely limited not only for the individual but also for the community if resistance occurs to a high degree among treated patients.

\section{CREATING DURABLE ANTIRETROVIRAL REGIMENS/PROGRAMS IN CHINA}

Successful treatment strategies are based on the concept of durability. A durable regimen can prevent the emergence of resistance, promote health, decrease the complexities of care, and save money in total health care costs. Durable regimens can be created with prudent, intelligent use of the antiretroviral agents currently available. Select- 
Tab. 1 Potential First Line Reverse Transcriptase Inhibitor based Regimens

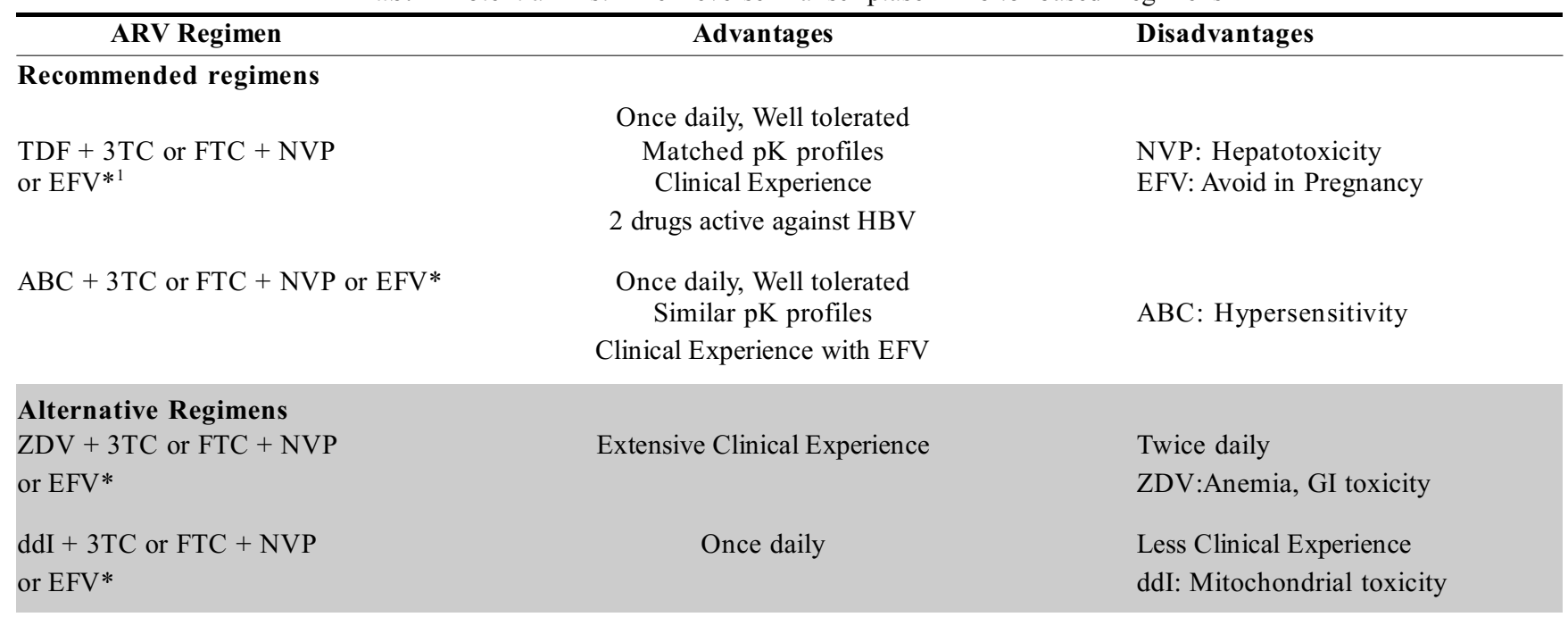

\section{Regimens to Consider - More Evidence Required}

ZDV/ABC/3TC/TDF

$\mathrm{ZDV} / \mathrm{ABC} / 3 \mathrm{TC}$

ZDV/3TC/TDF

\section{Well tolerated}

Few drug interactions

No NNRTI

2 drugs active against $\mathrm{HBV}$

Well tolerated

Few drug interactions

No NNRTI
Less Clinical Experience

Twice daily

ZDV:Anemia, GI toxicity

ABC: hypersensitivity

Lower Efficacy at Viral Loads >

100,000 and Compared to EFV

Regimen

Twice daily

ZDV:Anemia, GI toxicity

$\mathrm{ABC}$ : hypersensitivity

Less Clinical Experience

Twice daily

ZDV:Anemia, GI toxicity

\section{Regimens Not Recommended Due to Toxicity or High Failure Rates}

$\begin{array}{ll}\mathrm{d} 4 \mathrm{~T} / 3 \mathrm{TC}+\mathrm{NVP} \text { or EFV* } & \begin{array}{l}\text { Inexpensive, Well tolerated in } \\ \text { short term,Clinical Experience }\end{array}\end{array}$

$\mathrm{d} 4 \mathrm{~T}$ : long term mitochondrial short term,Clinical Experience toxicities and lipodystrophy

d4T/ddI/NVP

Once Daily

Additive toxicities of ddI and d4T

TDF/ddI/NVP

High failure rates

ddI toxcities

$\mathrm{TDF} / \mathrm{ABC} / 3 \mathrm{TC}$

$\mathrm{TDF} / \mathrm{ddI} / 3 \mathrm{TC}$

Once Daily

ddI/ABC/3TC

*Nevirapine has restrictions on its use due to hepatotoxicity noted in women with CD4 $>250$ and men with CD4 $>400$. Efavirenz is contraindicated in pregnancy and in women of child bearing age.

${ }^{1} \mathrm{TDF}$ based regimen is preferred in China due to the high prevlence of HBV and the desire to avoid HBV monotherapy. Efavirenz is favored due to the low fertility rate and the lack of CD4 count restrictions for use as seen with NVP.

ing treatment regimens and delivery strategies prior to validating durability of the selected regimen in the specific patient population could have long term serious public health consequences. For example, recent clinical trials found that certain combinations of reverse transcriptase inhibitors, ddI/ABC/3TC, ddI/TDF/3TC or ABC/TDF/3TC, had unacceptable high rates of failure only after they were used clinically [41, 42]. 
Central to the principles of optimizing durable ART is the attainment of maximal viral suppression, a viral load less than 50 HIV RNA copies/ml. Only clinically proven regimens of 3 or more primary ARV drugs should be used. Drugs with high mutation thresholds should be included. This is particularly important when considering the use of 3TC and NNRTI drugs which are very vulnerable to loss of activity due to an occurrence of a single point mutation. Because patients interrupt medication due to illness and non-adherence, it is also important to use medications with similar $\mathrm{pK}$ profiles. However as important as the regimen choice is, the care delivery structure used to deliver ART is equally as important. Treatment adherence is paramount to durable viral suppression.

This is particularly important during the induction of viral control and periods when virus is detectable. Data is emerging that events during the initial induction of viral control will determine durability limitations not recognized until 1-2 years later. Thus programs like our own are developing intensive treatment preparation strategies to adequately prepare patients for treatment. ARV treatment preparation is then coupled with intensive treatment support especially during the first 3 months after treatment initiation to include a menu of observational ARV delivery strategies. Both treatment preparation and treatment support proactively incorporate family and community involvement into HIV care programs. All of this is intended to give the patient needed support to ensure adherence particularly when patients are first placed on ART. Given that much remains to be learned as relates to alternative ARV delivery models, it is important to create and validate new models for antiretroviral treatment strategies for different settings and populations.

Choice of the optimal regimen to be used in different clinical settings of China remains to be defined. Despite this, the selection of initial first line ARV regimen choice is required. Today most programs have selected RTI based regimens for scale-up and held boosted PI based regimens for special situations and clinical failure of initial treatment. Initially, China explored the use of ZDV, ddI and NVP as well as d4T, ddI and NVP. Both these choices were associated with high treatment failure rates as noted above'. Tab. 1 outlines the advantages and disadvantages of potential RTI based treatment regimens and recommendations for China taking into consideration important concomitant issues such as the high prevalence of HBV and the low fertility rate. With the choice of potent regimens consistent with the principles highlighted above and intensive treatment preparation and support especially during the first 3 months of treatment initiation, durable antiretroviral suppression should become the rule.

\section{OPPORTUNITIES FOR SUCCESS}

Today a person diagnosed with HIV infection in the US or Western Europe can anticipate living a natural life time. China's implementation of a national ARV treatment program has the potential to accomplish this same feat in China. However, the key to this accomplishment is the successful introduction of durable, scalable, sustainable antiretroviral therapy.

ART has major limitations which have been appreciated over the last 15 years and has caused HIV therapy to become more complex and costly over time in the developed world. Successful strategies to bring HIV therapies for all regions and populations in China need to be developed in context of the complexities of using ARV in each region with variable health care infrastructures, limited monitoring capabilities, different opportunistic infections and nutritional deficiencies, and different levels of HIV education and stigmatizations. Local solutions need to be developed, implemented and embraced. What works well in the US may not work well in China. Available data from African and Caribbean populations strongly suggest that time to development of clinical outcome, after HIV infection, is significantly compressed compared to US and European populations, and that differences in the health care delivery systems account for only a part of this accelerated disease process. These therapeutic programs and approaches must also be carefully considered and anchored in what is known about the pathogenesis of HIV/AIDS in such populations and not simply the blanket application of known US and Europe therapeutic strategies [43-46].

China has begun a process of evaluating the performance of local programs through systematic monitoring and evaluation of treatment outcomes. This will allow regimens and programs that work to be expanded, and programs with high failure rates to be eliminated. "Successful" ART should not merely count the number of patients treated, but rather programs must be measured by treatment outcomes and their consequences on the society. This will be important to ensure that programs that are ultimately recognized as failures in 5-10 years, do not get exponentially expanded prematurely. However, if demonstrated success is linked to additional resources, and these programs are brought to scale as rapidly as possible, the integration of science, medicine, industry and public policy in to delivering ART in China will allow China to successfully confront its AIDS epidemic early and prevent its tragic consequences.

\section{REFERENCES}

1 Zhang FJ, Pan J, Yu L, Wen Y, Zhao Y. Current Progress of China's Free ART Program, Cell Research 2005; 15:877-82.

2 Robbins GK, DeGruttola V, Shafer RW, et al. Comparison of 
Sequential Three-Drug Regimens as Initial Therapy for HIV-1 Infection. NEJM, 2003; 349:2293-303.

3 Tavel JA, Miller KD, Masur H. Guide to major clinical trials of antiretroviral therapy in human immunodeficiency virus-infected patients: Protease inhibitors, nonnucleosdie reverse transcriptase inhibitors, and nucleotide reverse transcriptase inhibitors. CID, 1999; 28:643-76.

4 Truchis P, Force G, Welker Y, et al. An open-label study to evaluate efficacy and safety of a quadruple combination therapy without protease inhibitor (Combivir+abacavir+efavirenz) in antiretroviral therapy naïve adults (CNAF3008) In: XIII International AIDS Conference; July 9-14, 2001; Durbin, SA. Abstract 3208

5 Staszewski S,Gallant JE,Pozniak AL, et al. Efficacy and safety of tenofovir DF (TDF) versus stavudine (d4T) when used in combination with lamivudine and efavirenz in antiretroviral naive patients: 96 week preliminary interim results. In: $10^{\text {th }}$ Conference on Retroviruses and Opportunistic Infections; Feb. 10-14, 2003; Boston, MA. Abstract 564b

6 Lucas GM, Chaisson RE, Moore RD Survival in an Urban HIV1 Clinic in the Era of Highly Active Antiretroviral therapy: A 5Year Cohort Study. JAIDS 2003; 33:321-8.

7 Little SJ, Holte S, Routy JP, et al. Antiretroviral-drug resistance among patients recently infected with HIV. N Engl J Med 2002; 347:385-94.

8 Richman DD, Morton SC, Wrin T, et al. The prevalence of antiretroviral drug resistance in the United States. AIDS 2004; 18:1393-401.

9 Li JY, Li H, Li, L, et al. Prevalence and evolution of drug resistance HIV-1 variants in Henan, China. Cell Research 2005; 15: 843-9.

10 Lucas GM, Weidle PJ, Hader S, Moore RD. Directly administered antiretroviral therapy in an urban methadone maintenance clinic: a nonrandomized comparative study. Clin Infect Dis. 2004 Jun 1; 38 Suppl 5:S409-13

11 Fischl M, Rodriguez A, Scerpella E, Monroig R, Thompson L and Rechtine D. Impact of Directly Observed Therapy on Outcomes in HIV Clinical Trials. $7^{\text {th }}$ Conference on Retroviruses and Opportunistic Infections, San Francisco, CA, January 30February 2, 2000, Abstract 71.

12 Koenig SP, Leandre F, Farmer PE. Scaling-up HIV treatment programmes in resource-limited settings: the rural Haiti experience. AIDS. 2004 Jun;18 Suppl 3:S21-5.

13 Scaling-up antiretroviral therapy in resource-limited settings. Geneva: World Health Organization 2002.

14 Accelerating access to care. Geneva: Joint United Nations Programme on HIV/AIDS 2002.

15 Brindeiro RM, Diaz RS, Sabino EC et al. Brazilian Network for HIV Drug Resistance Surveillance (HIV-BResNET): a survey of chronically infected individual. AIDS. 2003, 17:1063-9

16 Tanuri A. Brazilian Network of HIV Drug Resistance Surveillance (HIV-BResNet): Results from the National survey Done in 2001. Presented at Global HIV/AIDS Program of the World Bank Group. Provision of ARV Therapy in Resource Limited Settings: The Challenges of Drug Resistance and Adherence. Washington D.C. June 17-18 2003.

17 McMahon C. 2nations unite on AIDS care-Argentina, Brazil vow to make generic drugs. Chicago Tribune, August 25, 2005, 4.
18 Chun TW, Carruth L, Finzi D, et al. Quantification of latent tissue reservoirs and total body viral load in HIV-1 infection. Nature 1997; 387:183-7.

19 Chun TW, Stuyver L, Mizell SB, et al. Presence of an inducible HIV-1 latent reservoir during highly active antiretroviral therapy. Proc Natl Acad Sci U S A; 1997; 94:13193-7.

20 Finzi D, Hermankova M, Pierson T, et al. Identification of a Reservoir for HIV-1 in Patients on Highly active Antiretroviral Therapy. Science 1997; 278:1295-300.

21 Wong JK, Hezareh M, Gunthard HF, et al. Recovery of Replication-Competent HIV Despite Prolonged Suppression of Plasma Viremia. Science 1997; 278:1291-4.

22 Chun TW, Fauci AS. Latent reservoirs of HIV: Obstacles to the eradication of virus. Proc Natl Acad Sci U S A 1999; 96:1095861.

23 Hogg RS,Yip B, Wood E, et al. Diminished Effectiveness of Antiretroviral Therapy among Patients Initiating Therapy with CD4+ Cell Counts Below 200/mm3. In: 8th Conference on Retrovirus and Opportunistic Infections; Feb 4-8, 2001; Chicago, IL. Abstract 342.

24 Kaplan J, Hanson D, Karon J, et al. Late Initiation of Antiretroviral Therapy (at CD4+Lymphocyte Count $<200$ Cells/ $\mathrm{mL}$ ) Is Associated with Increased Risk of Death. In: 8th Conference on Retrovirus and Opportunistic Infections; Feb 4-8, 2001; Chicago, IL. Abstract 520.

25 Opravil M, Ledergerber B, Furrer H, et al. Clinical Benefit of Early Initiation of HAART in Patients with Asymptomatic HIV Infection and CD4Counts $>350 / \mathrm{mm} 3$. In: 8 th Conference on Retrovirus and Opportunistic Infections; Feb 4-8, 2001; Chicago, IL. Abstract LB6.

26 Sterling TR, Chaisson RE, Bartlett JG, et al. CD4+Lymphocyte Level Is Better than HIV-1 Plasma Viral Load in Determining When To Initiate HAART. . In: 8th Conference on Retrovirus and Opportunistic Infections; Feb 4-8, 2001; Chicago, IL. Abstract 519 .

27 Medina DJ, Tsai CH, Hsiung GD, Cheng YC. Comparison of mitochondrial morphology, mitochondrial DNA content, and cell viability in cultured cells treated with three anti-human immunodeficiency virus dideoxynucleosides. Antimicrob Agents Chemother 1994; 38:1824-8.

28 Carr A, Saeras K, Burton S, et al. A syndrome of peripheral lipodystrophy, hyperlipidemia, and insulin resistance in patients receiving HIV protease inhibitors. AIDS 1998; 12:F51-58.

29 Viramune package insert. Ridgefield, CT: Boehringer Ingelheim Pharmaceuticals, Inc., revised Sept. 4, 2003

30 Brinkman K, ter Hofstede HJ, Burger DM, Smeitink JA, Koopmans PP. Adverse effects of reverse transcriptase inhibitors: mitochondrial toxicity as common pathway. AIDS 1998; 12: $1735-44$

31 Copeland WC, Chen MS, Wang TSF. Human DNA polymerases alpha and beta are able to incorporate anti-HIV deoxynucleotides into DNA. J Biol Chem 1992; 267:21459-64.

32 Fortgang IS, Belitsos PC, Chaisson RE, Moore RD. Hepatomegaly and steatosis in HIV-infected patients receiving nucleoside analog antiretroviral therapy. Am J Gastroenterol 1995; 90:14336.

33 Sundar K, Suarez M, Banogon PE, Shapiro JM. Zidovudineinduced fatal lactic acidosis and hepatic failure in patients with acquired immunodeficiency syndrome: Report of two patients 
and review of the literature. Crit Care Med 1997; 25:1425-30.

34 Paterson DL, Swindells S, Mohr J, et al. Adherence to protease inhibitor therapy and outcomes in patients with HIV infection. Ann Intern Med 2000; 133:21-30.

35 Chaisson RE, Keruly JC, Moore RD. Association of initaial CD4 cell count and viral load with response to highly active antiretroviral therapy. JAMA 2000; 284:3128-3129.

36 Casado JL, Perez Elias MI, Antela A, et al. Predictors of longterm response to protease inhibitor therapy in a cohort of HIV infected patient. AIDS 1998; 12:F131-51

37 Deeks SG, Hecht FM, Swanson M, et al. HIV RNA and CD4 cell count response to protease inhibitor therapy in an urban AIDS clinic: Response to both initial and salvage therapy. AIDS 1999; 13:F35-43.

38 Lucas GM, Chaisson RE, Moore RD. Highly active antiretroviral therapy in a large urban clinic: Risk factors for virologic failure and adverse drug reactions. Ann Intern Med 1999; 131:81-7.

39 Ledergerber B, Egger M, Opravil M, et al. Clinical progression and virological failure on highly active antiretroviral therapy in HIV-1 patients: A prospective cohort study. Swiss HIV Cohort Study. Lancet, 1999; 353:863-8.

40 Eshleman SH, Mracna M, Guay G, et al. Selection of Nevirapine Resistance (NVPR) Mutations in Ugandan Women and Infants Receiving NVP Prophylaxis To Prevent HIV-1 Vertical Transmission (HIVNET-012). In: 8th Conference on Retrovirus and
Opportunistic Infections; Feb 4-8, 2001; Chicago, IL. Abstract 516.

41 Farthing C, Khanlou H, Yeh V. Early virologic failure in a pilot study evaluating the efficacy of once daily abacavir, lamivudine, and tenofovir DF in treatment naïve HIV-infected patients.AIDS Patient Care STDS 2005; 19:135-40.

42 Gallant JE, Rodriguez AE, Weinberg WG, et al. Early virologic non-response to tenofovir, abacavir, and lamivudine in HIV infected antiretroviral-naïve subjects. J Infect Dis 2005; 192:192130.

43 Kanki P, Hamel D, Sankale L, et al. HIV type 1 subtypes differ in disease progession. J. Infect Dis 1999; 179:68-73.

44 DeWit S, Boulme R, Poll B, Schmit JC, Clumeck N. Viral load and CD4 response to PI containing Regimen in B versus non-B treatment-naïve HIV-1 patients. $2^{\text {nd }}$ IAS Conference on HIV Pathogenesis and Treatment. Paris, July 13-16 2002 (abstract 25)

45 Morgan D, Mahe C, Mayanja B, Whitworth J. Progression to symptomatic disease in people infected with HIV-1 in rural Uganda: prospective cohort study: BMJ 2002; 324:193-6.

46 Constantine NT, Sill AM, Jack N, Kreisel K, Edwards J, Cafarella T, Smith H, Bartholomew C, Cleghorn FR, Blattner WA. Improved Classification of Recent HIV-1 Infection by Employing a Two-stage Sensitive/Less-sensitive Test Strategy, J Acquir Immune Defic Syndr. 2003; 32:94-103. 